\title{
11. Conclusio
}

Bürgerwehren in Deutschland sind ein diverses Phänomen. Von Nachbar"innen, die sich zu abendlichen Spaziergängen durch ihr Viertel verabreden, um nach Einbrechern Ausschau zu halten, bis hin zu organisierten Rechtsextremen, die die Struktur einer Bürgerwehr politisch nutzen, um Dominanz und Kontrolle im öffentlichen Raum auszuüben und "Angstzonen ${ }^{1}$ zu kreieren die Bandbreite ist groß. Um dieser Diversität gerecht zu werden, wurde in dieser Studie eine Unterteilung der Bürgerwehren in drei Typen vorgenommen, von denen dann jeweils eine Bürgerwehr in qualitativen Einzelfallstudien ethnografisch untersucht wurde. Anleitend war die Fragestellung: Sind Bürgerwehren Akteure im Versicherheitlichungsprozess?

Die Bürgerwehr Harzberg zählt zum Typ der Institutionalisierten Bürgerwehren (Typ I). Sie gründete sich nach einer Einbruchsserie im Ort, um das Eigentum der Bürger“innen zu schützen. Für Typ II (Autonome Bürgerwehren) wurde die Bürgerwehr in Weißensee ausgewählt. Ihre Mitglieder geben an, mit ihren Patrouillen Frauen vor sexuellen Übergriffen, die von rassifizierten Personen verübt würden, schützen zu wollen. Der dritte Typ (Aktivistische Bürgerwehren) wird aus bestehenden politischen Gruppen heraus gegründet, um politische Machtgewinne zu erzielen. Hier wurde die SchutzzonenKampagne der NPD ausgewählt und beispielhaft die Schutzzonen-Gruppe in Berlin Mitte untersucht.

Die Einzelfallstudien haben gezeigt, dass sich die Bürgerwehren in Harzberg und Weißensee sowie die Schutzzonen-Kampagne der NPD als Akteure der Versicherheitlichung im Sinne der Kopenhagener Schule beschreiben

1 Vgl. zum Konzept der »Angstzonen« Döring, Uta (2008): Angstzonen. Rechtsdominierte Orte aus medialer und lokaler Perspektive. Wiesbaden: Verlag für Sozialwissenschaften. S. $141 \mathrm{ff}$. 
lassen. Ihnen gelingt es, Bedrohungslagen zu etablieren und ihre Patrouillen als extraordinary measure zu setzen, die die Sicherheit wiederherstellen sollen. Die Versicherheitlichungsprozesse weisen dabei bei den untersuchten Bürgerwehren jeweils eigene Dynamiken auf, sind aber in ihrer Struktur vergleichbar. So bilden in allen untersuchten Fällen Unsicherheitswahrnehmungen die Grundlage für die Gründung der Bürgerwehren. Die Referenzobjekte, auf die sich die Unsicherheitsgefühle beziehen, sind in den einzelnen Bürgerwehren unterschiedlich stark ausgeprägt (vgl. Kapitel 9), die von den Bürgerwehren beschriebenen Bedrohungslagen weisen jedoch alle eine Unterscheidung zwischen einem sicheren »Innen« auf, das vor einem gefährlichen »Außen« beschützt werden müsse. Die Grenzziehung zwischen innen und außen verläuft entlang der Unterscheidung »einheimisch«/»fremd«. Von denjenigen, die zur von den Bürgerwehren beschriebenen Gemeinschaft gehören, geht in den Augen der Bürgerwehren keine Gefahr aus, sie sollen beschützt werden. Die Gefahr wird in allen Fällen in einem bedrohlichen Außen verortet.

Die Unterscheidung zwischen schützenswert und bedrohlich trennt dabei Personen, die Zugang zu Privilegien haben, von Menschen, die aufgrund ihrer Herkunft von diesen Privilegien ausgeschlossen sind. Durch innereuropäische Grenzöffnungen und globale Migrationsbewegungen ist letztere Gruppe physisch präsent geworden und wird von den Bürgerwehren als Bedrohung empfunden. Die Bürgerwehren sind ein Projekt zur Verteidigung von Privilegien, zur Verteidigung eines gesellschaftlichen Status', der aus Eigentum, Kontrolle über die Sexualität von Frauen und kultureller Hegemonie besteht.

In Harzberg ist es die Angst vor »osteuropäischen Banden«, die »wie die Heuschrecken« über Harzberg herfallen und stehlen würden, was sich die Bürger"innen von Harzberg aufgebaut hätten. In Weißensee sind die »Fremden«, Geflüchtete, die im Zuge der Migrationskrise 2015/16 nach Deutschland kamen und angeblich deutsche Frauen sexuell belästigen würden. Die Schutzzone in Berlin Mitte will »Deutsche« und Tourist"innen vor einer kriminellen "Zigeunerbande« beschützen. Die Bürgerwehren knüpfen mit den von ihnen wahrgenommenen Bedrohungslagen an stark emotionalisierte gesellschaftliche Unsicherheiten an, die bereits in der medialen Debatte politisiert sind. Dabei berufen sie sich auf eine wahrgenommene Lücke, die durch Stellenabbau bei der Polizei entstehe, und implementieren ihre Patrouillen als extraordinary measure, um diese Lücke zu schließen.

Bürgerwehren als Akteure von Versicherheitlichungen zu beschreiben, bedeutet, eine Theorie der Internationalen Beziehungen auf einen lokalen 
Kontext anzuwenden. Diesem Transfer wohnt ein gewisses Spannungsverhältnis inne, das sich jedoch als produktiv für eine Weiterentwicklung der Theorie erwiesen hat. So haben die Mitglieder der Bürgerwehren, also die Sprecher im Versicherheitlichungsprozess, keine position of authority innerhalb der lokalen Gemeinschaft.

Es gelingt ihnen dennoch, die Patrouillen als eine extraordinary measure zu implementieren. Es gelingt ihnen, weil sie sich eigenmächtig entschließen, den öffentlichen Raum zu patrouillieren. Oder, wie ein SchutzzonenMitglied sagt: »Wir machen es einfach, auch wenn's der Polizei (lacht) nicht passt ${ }^{2}$ Der Sprechakt fällt hier zusammen mit der Implementierung der $e x-$ traordinary measure. In dem Moment, wo die Gruppe als Bürgerwehr auf Patrouille geht, formuliert sie die Bedrohungslage und setzt die außerordentliche Sicherheitsmaßnahme, um ihr zu begegnen. Mit dem Akt der Gründung erlangen die Mitglieder zudem die Autorität, die die Theorie der Versicherheitlichung von der Sprecherposition verlangt.

Jedoch ist die Versicherheitlichung im Fall der Bürgerwehren unter diesen Umständen eine instabile, die die Akteure durch eine permanente ReFormulierung der Bedrohungslage zu stabilisieren versuchen. Die Fragilität der Versicherheitlichungen im Fall von Bürgerwehren vermag die Flüchtigkeit des Phänomens zu erklären und zeigt, dass eine Weiterentwicklung der Theorie, im Sinne einer Berücksichtigung subalterner Versicherheitlichungsakteure, fragile Prozesse der Versicherheitlichung erklären könnte.

Die Fragilität des Versicherheitlichungsprozesses und die Flüchtigkeit des Phänomens bedeuten jedoch nicht, dass die gesellschaftlichen Auswirkungen von Bürgerwehren geringer sind. Im Gegenteil: Diese fragilen Versicherheitlichungen auf der Mikroebene hinterlassen jede für sich ein Echo, das die Unsicherheitswahrnehmungen der lokalen Bevölkerung nachhaltig beeinflusst. In ganz Deutschland gründeten sich in den letzten Jahren hunderte Bürgerwehren. ${ }^{3}$ So entsteht ein Flickenteppich aus Mikroversicherheitlichungen auf lokaler Ebene, ein Echoraum aus sich gegenseitig überlappenden Bedrohungslagen, der die Basis für Versicherheitlichungen auf der Makroebene bildet.

2 Transkript Schutzzone Hofburg I, Zeile 149.

3 Anika Hoffmann kommt in ihrer quantitativen Erhebung von Bürgerwehrgruppen auf Facebook auf 456 Gruppen, die am Tag der Erhebung im Dezember 2016 bestanden. Vgl. Hoffmann, Anika (2019): Bürgerwehren und ihre Bedeutung im öffentlichen Raum. Abweichendes Verhalten als kriminologisch relevantes Phänomen. Wiesbaden: VS Verlag. S. 61. 
So lässt sich mit einer Untersuchung von Mikroversicherheitlichungen in lokalen Kontexten eine Lücke in der Theorie der Versicherheitlichung schließen. Denn Unsicherheitswahrnehmungen werden in der Theorie oft als gegeben vorausgesetzt. Mit einem Blick auf die Mikroebene kann aufgezeigt werden, wie gesamtgesellschaftliche Unsicherheitswahrnehmungen entstehen und Bedrohungslagen auf gesamtgesellschaftlicher Ebene an bereits mikroversicherheitlichte Themen anknüpfen, die im Echoraum der Mikroversicherheitlichungen schwingen.

Dieses vielstimmige Echo ist umso wirkungsvoller, wenn mit den einzelnen Unsicherheitswahrnehmungen an dasselbe Narrativ angeknüpft wird. Beispielsweise wurde das Narrativ »des übergriffigen Fremden«, vor dem »unsere Frauen« beschützt werden müssten, und auf das die Bürgerwehr in Weißensee sich bezieht, von zahlreichen Bürgerwehren, die sich während der medialen Debatte über die Ereignisse der Silvesternacht 2015/16 in Köln gründeten, aufgegriffen. Aber auch unterschiedliche Bedrohungslagen verstärken ein generelles diffuses Unsicherheitsgefühl in der Bevölkerung und erleichtern ein sicherheitspolitisches Aufrüsten der Gesellschaft. Bürgerwehren fungieren hier als Motor, sie erzeugen Druck, auf den die Politik reagiert, beispielsweise mit einer restriktiveren Asylpolitik.

Am Beispiel des Bedrohungsnarrativs des »übergriffigen Fremden« zeigt sich, dass der Versicherheitlichungsprozess im Fall von Bürgerwehren stark vergeschlechtlicht ist. Die Vergeschlechtlichung von Versicherheitlichungsprozessen ist bisher kaum untersucht worden. ${ }^{4}$ Es existieren zwar Studien über die Versicherheitlichung von Phänomenen, die vergeschlechtlicht sind, nicht jedoch über die Dynamik, die die soziale Kategorie Geschlecht im Versicherheitlichungsprozesss selbst entwickelt.

Im Fall der Bürgerwehren wirkt Geschlecht als Katalysator des Versicherheitlichungsprozesses. Fast alle Mitglieder der untersuchten Bürgerwehren sind männlich. Sie verkörpern eine marginalisierte Männlichkeit ${ }^{5}$, die sich in spannungsreichen und fragilen Männlichkeitsidentitäten äußert. Durch die Teilnahme an den Bürgerwehren wird ein als entmännlichend empfundenes

4 Vgl. hierzu die Kritik von Hansen, Lene (2000): The Little Mermaid's Silent Security Dilemma and the Absence of Cender in the Copenhagen School. Millennium. Vol. 29, Nr. 2, Juni 2000. S. 285-306.

5 Vgl. hierzu Connell, Raewyn (2015): Der gemachte Mann, Geschlecht und Gesellschaft. Konstruktion und Krise von Männlichkeiten. 4. erw. Aufl., Wiesbaden: Springer Fachmedien. 
potenzielles zum-Opfer-werden präventiv abgewehrt. Die Rolle des Beschützers ermöglicht die Inszenierung eines Habitus der Stärke, das mit einer (visuellen) Betonung von Muskelkraft und Körpermasse einher geht.

Zudem existiert innerhalb der Gruppen eine ausgeprägte Geschlechterordnung, die in allen drei Bürgerwehren dieser Studie nahezu identisch ist. Die Männer sind aktiv, begeben sich in Gefahr, sind die Beschützer, wohingegen die wenigen Frauen, die sich überhaupt in Bürgerwehren einbringen, meist für eine logistische und emotionale Unterstützung der Männer zuständig sind. ${ }^{6}$ Hier ermöglicht die Bürgerwehr den männlichen Mitgliedern Autorität und Überlegenheit - Eigenschaften, die einer marginalisierten Männlichkeit normalerweise schwer zugänglich sind. Die Geschlechterordnung innerhalb der Bürgerwehr sowie die Rolle des Beschützers ermöglichen es den Männern, ihre fragilen Männlichkeitsidentitäten zu stabilisieren. Um die stabilisierende Wirkung aufrecht zu erhalten, die die Bürgerwehrpatrouillen auf die Männlichkeitsidentitäten der Mitglieder haben, müssen die Bedrohungslagen ständig reformuliert werden und der Akt des Beschützens immer wieder neu inszeniert werden. Hier zeigt sich, dass Geschlecht als Beschleuniger des Versicherheitlichungsprozesses wirkt sowie die Dauer der Versicherheitlichung beeinflusst.

Im Fall der Bürgerwehr in Weißensee ist der Versicherheitlichungsprozess in doppelter Weise vergeschlechtlicht. Nicht nur wirkt das Geschlecht der Sprecher der Versicherheitlichung auf die Dynamik des Prozesses, auch das Referenzobjekt ist vergeschlechtlicht. Diese doppelte Vergeschlechtlichung ermöglicht es den Männern, sich als Beschützer von Frauen zu inszenieren. Ein Bedürfnis nach Kontrolle der weiblichen Sexualität wirkt hier mit dem rassistischen Bild des »übergriffigen Fremden« zusammen und treibt den Versicherheitlichungsprozess voran.

In ihrer Rolle als Beschützer übernehmen die Bürgerwehren eigenmächtig polizeiliche Aufgaben. Sie nehmen eine Lücke in der Ausführung der staatlichen Aufgabe, Schutz zu gewährleisten, wahr und beanspruchen für sich, mit ihren Patrouillen die Sicherheit wiederherzustellen, die der Staat nicht zu gewährleisten im Stande sei. Damit untergraben Bürgerwehren das staatliche Gewaltmonopol und stellen den Staat als Sicherheitsgaranten infrage. 
Die untersuchten Gruppen kritisieren darüber hinaus jedoch auch den Umfang der Befugnisse, die den Polizeikräften für ihre Arbeit zur Verfügung stehen. Sie positionieren sich damit als Akteur, der eigene Maßstäbe anlegt, mit denen Sicherheit hergestellt werden soll.

Selbst wenn sich die Mitglieder dabei im Rahmen des Legalen bewegen, selbst wenn sie sich mit ihrem Auftreten als Bürgerwehr an die Gesetze halten, also lediglich vom Jedermannsrecht Gebrauch machen, ihr Handlungsspielraum ist dennoch groß. Die Einzelfallstudien haben gezeigt, dass die politischen Meinungen der Mitglieder ihr Agieren im öffentlichen Raum beeinflussen.

Die Bürgerwehren verorten die Täter*innen nahezu ausschließlich in der Gruppe der als fremd wahrgenommenen Personen. Diese einseitige Projektion von Gefahr auf Menschen, die durch ihr Aussehen ${ }^{7}$ beschrieben werden, verletzt des Grundprinzip des demokratischen Rechtsstaats: Vor der Bürgerwehr ist nicht jede* $r$ gleich. Die Bürgerwehren von Typ II und III sind zudem Ausdruck von Machtgewinnen durch Raumgewinne und Normalitätsgewinne der extremen Rechten. Im Fall der Bürgerwehr des Typs III geschieht dies im Rahmen einer von außen gesteuerten, groß angelegten Strategie der NPD.

Die Gruppen von Typ II und III haben mit ihrer Präsenz im öffentlichen Raum eine politische Funktion. So sind diskursive Verschiebungen in der öffentlichen Debatte seit der Migrationskrise 2015/16 und eine breitere Akzeptanz neurechter Narrative zu beobachten. Gleichzeitig ist eine Zunahme von rechten Gewalttaten zu verzeichnen. Im Zusammenspiel zwischen diesen sprachlichen Verschiebungen und den wachsenden Zahlen rassistisch motivierter Gewalttaten seit 2016 übernehmen Bürgerwehren eine Scharnierfunktion. In ihrem Auftreten kristallisiert sich die rassistische Stimmungslage, sie erreicht die praktische Handlungsebene.

Bürgerwehren in Deutschland sind ein relativ neues Forschungsfeld. Die Häufung in den letzten Jahren sowie die politische Brisanz des Phänomens zeigen, dass hier weitere Forschung unabdingbar ist. So mangelt es zunächst an quantitativen Untersuchungen, die regionale Verteilung, Überlebensdauer und Gründungshäufungen aufzeigen. Ebenfalls kaum erforscht nen als sexuell übergriffig. Und die NPD-Bürgerwehr knüpft mit ihrer Täterbeschreibung an das Narrativ der »kriminellen Ausländer«, insbesondre der klauenden »Zigeunerbanden«an. 
sind staatliche Sicherheitskonzepte, die unqualifizierte Bürger*innen in die Polizeiarbeit einbinden. Hier wäre insbesondere eine Untersuchung der politischen Haltungen der Hilfspolizist"innen relevant. So ruft beispielsweise die Schutzzonen-Kampagne der NPD ihre Sympathisant"innen dazu auf, sich in diesem Rahmen in die Polizeiarbeit einzubringen. ${ }^{8}$

Auch in der Rechtswissenschaft besteht Nachholbedarf. Der bundesweit uneinheitliche Umgang der Polizei mit den NPD-Bürgerwehren zeigt, dass der Staat keine Handhabe für diesen Angriff auf sein Gewaltmonopol zu haben scheint. Auch existieren bisher keine Urteile zu kollektiven Tätigkeiten von Bürgerwehren, es gibt lediglich Einzelverurteilungen bei konkreten Gesetzesverstößen. ${ }^{9}$ Nicht zuletzt bedarf es einer weiteren Untersuchung der in dieser Arbeit beschriebenen Strategie rechtsextremer Akteure, durch das eigenmächtige Übernehmen staatlicher Aufgaben sowie durch die gezielte Einnahme des öffentlichen Raums Machtgewinne zu erzielen.

8 Vgl. Facebook-Post der Schutzzonen-Kampagne. Online unter: https://www.facebook. com/schutzzone/posts/435401507011201/ (Zugriff am 2.1.2021)

9 Vgl. Hoffmann, Anika (2019): Bürgerwehren und ihre Bedeutung im öffentlichen Raum. Abweichendes Verhalten als kriminologisch relevantes Phänomen. Wiesbaden: VS Verlag. S. 15. 
